\title{
$(n, p)$ reactions contribution in spherical proton recoil proportional counter responses
}

\author{
R. Babut ${ }^{a}$ and V. Gressier \\ ${ }^{a}$ ARPHYTECH, \\ 7, rue de la Croix Martre, 91120 Palaiseau, France \\ ${ }^{b}$ IRSN, \\ Cadarache, 13108 St-Paul-lez-Durance, France \\ E-mail: vincent.gressierdirsn.fr
}

ABSTRACT: Proton recoil proportional counters are used in several metrological institutes as reference neutron spectrometers within the energy range from a few tenth of $\mathrm{keV}$ up to a few $\mathrm{MeV}$. When such counters are used to determine the low energy range of neutron fields with energies above $5 \mathrm{MeV}$, protons generated by $(\mathrm{n}, \mathrm{p})$ reactions within the stainless steel counter walls will add their contribution to the proton recoil process. In this work, this contribution has been estimated in the calculated response of the counter, using MCNPX Monte Carlo code. The validation of the calculated response has been performed at several AMANDE mono-energetic neutron fields.

KEYWORDS: Detector modelling and simulations I (interaction of radiation with matter, interaction of photons with matter, interaction of hadrons with matter, etc); Gaseous detectors; Interaction of radiation with matter; Detector modelling and simulations II (electric fields, charge transport, multiplication and induction, pulse formation, electron emission, etc).

\footnotetext{
* Corresponding author.
} 


\section{Contents}

\begin{tabular}{|c|c|}
\hline 1. Introduction & 1 \\
\hline 2. Impact of the $(n, p)$ reactions on spherical proportional counter response & 2 \\
\hline $2.1(\mathrm{n}, \mathrm{p})$ reaction cross sections & 2 \\
\hline 2.2 SP2 spherical proportional counter response simulation & 2 \\
\hline 3. Comparisons between simulations and experimental data & 4 \\
\hline 3.1 Experimental set-up & 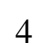 \\
\hline 3.2 Compariso & 4 \\
\hline
\end{tabular}

4. Conclusion and outlook

\section{Introduction}

Proton recoil proportional counters are used as reference spectrometers within the energy range of a few tenth of $\mathrm{keV}$ up to a few $\mathrm{MeV}$ by several metrological institutes [1] [2]. In the last years, the importance of the high energy neutron fields is increasing due to the development of fusion or medical facilities, high energy accelerators, etc. The recoil proton proportional counters can be used to determine precisely the energy distribution in the low energy part of these fields. However, the main drawback of these counters is their limited energy range. For neutron energies above the detector energy range, the corresponding recoil protons lose only a part of their energy in the detector gas. The contribution of these recoil protons is substracted using a downscattering correction [3], i.e the response of the detector to the neutron energy distribution above the counter energy range is subtracted to the experimental pulse height spectrum [4].

However, with high energy neutrons, $(n, p)$ reactions between the incident neutrons and the counter stainless steel wall compounds (Chromium, Iron, Nickel) create protons, which deposit also their energy in the gas of the detector. Generally, the energy threshold of these reactions is above a few $\mathrm{MeV}$. Whereas the elastic scattering neutron cross section on hydrogen decreases with the incident neutron energy, the $(n, p)$ reaction cross sections increase and induce a larger modification of the counter response energy distribution. This modification has to be taken into account in the simulation if these detectors are used to determine the neutron fluence energy distribution, below a few $\mathrm{MeV}$, of neutron fields with energies above several $\mathrm{MeV}$, in order to complete the neutron energy distribution obtained at higher energies by other detection systems as liquid scintillators.

In a first step, the response of a spherical proportional counter of SP2 type is calculated with the MCNPX Monte carlo code [5], with and without taking into account the (n,p) reactions into the detector walls. The calculation is performed at several neutron energies to determine the neutron energy above that the $(n, p)$ reactions contribution is no more negligible. The simulations are then compared to experimental data obtained at IRSN AMANDE facility [6] 


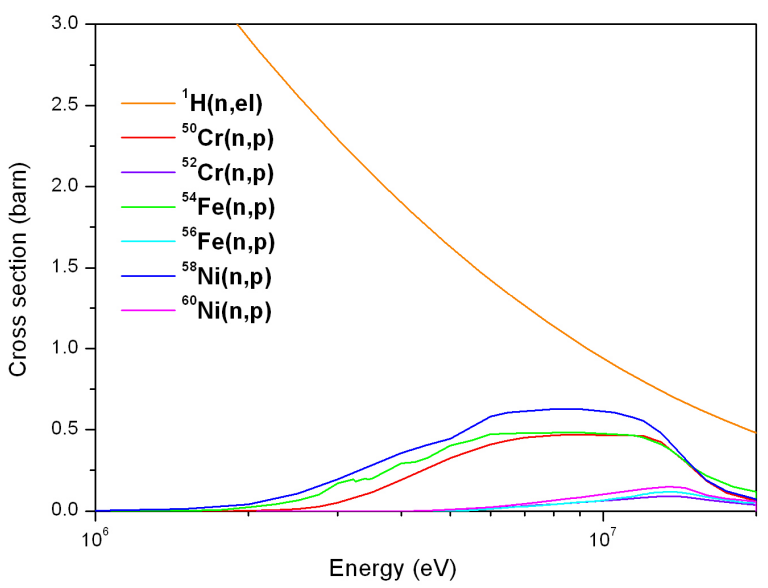

Figure 1. Comparison between the cross sections of the neutron elastic scattering reactions on hydrogen and the cross sections of the (n,p) reactions with the stainless steel elements [7].

\begin{tabular}{|l|l|l|l|}
\hline Reactions & $\mathrm{Q}_{\text {reaction }}(\mathrm{MeV})$ & $\mathrm{E}_{\text {threshold }}(\mathrm{MeV})$ & $\mathrm{E}_{\mathrm{p}} \max (\mathrm{MeV})$ \\
\hline${ }^{50} \mathrm{Cr}(\mathrm{n}, \mathrm{p})$ & -0.256 & 0.261 & $\approx 20$ \\
\hline${ }^{52} \mathrm{Cr}(\mathrm{n}, \mathrm{p})$ & -3.193 & 3.255 & $\approx 17$ \\
\hline${ }^{54} \mathrm{Fe}(\mathrm{n}, \mathrm{p})$ & 0.085 & no threshold & $\approx 20$ \\
\hline${ }^{56} \mathrm{Fe}(\mathrm{n}, \mathrm{p})$ & -2.913 & 2.965 & $\approx 17$ \\
\hline${ }^{58} \mathrm{Ni}(\mathrm{n}, \mathrm{p})$ & 0.401 & no threshold & $\approx 20$ \\
\hline${ }^{60} \mathrm{Ni}(\mathrm{n}, \mathrm{p})$ & -2.041 & 2.076 & $\approx 17$ \\
\hline
\end{tabular}

Table 1. (n,p) reaction thresholds for the main elements in the stainless steel and maximum proton energy for $20 \mathrm{MeV}$ incident neutrons.

\section{Impact of the $(n, p)$ reactions on spherical proportional counter response}

\section{$2.1(n, p)$ reaction cross sections}

The walls of the proton recoil proportional counters are typically made of stainless steel. When the incident neutron energy is higher than $2 \mathrm{MeV}$, the threshold of some $(\mathrm{n}, \mathrm{p})$ reactions with the elements present in the stainless steel given in table 1 can be reached and the corresponding reaction cross sections become not negligible compared to the elastic scattering cross section on hydrogen as shown in figure 1. In this figure all the data are issued from the ENDF-BVI database. As shown in table 1 the energy of the protons produced by the $(n, p)$ reactions can reach up to $10 \mathrm{MeV}$ and be then deposited at least partly in the detector gas.

\subsection{SP2 spherical proportional counter response simulation}

This study will focus on a $2 \mathrm{~cm}$ radius SP2 spherical proton recoil proportional counter filled with 3 atmosphere pure hydrogen. The voltage applied to the $25 \mu \mathrm{m}$ diameter wire anode is $1860 \mathrm{~V}$. The cathode is constituted by the spherical walls of the counter made of $0.5 \mathrm{~mm}$ thick stainless steel, most common wall thickness encountered for such counters.

The proton ranges in stainless steel for $10 \mathrm{MeV}, 15 \mathrm{MeV}$ and $20 \mathrm{MeV}$ protons are respectively of $0.25 \mathrm{~mm}, 0.50$ and $0.83 \mathrm{~mm}$. It means that if the maximum neutron energy is below $10 \mathrm{MeV}$, only neutron reactions inside the inside half layer of the wall generate protons able to reach the reactive gaseous volume of the counter. Therefore, for neutrons below 


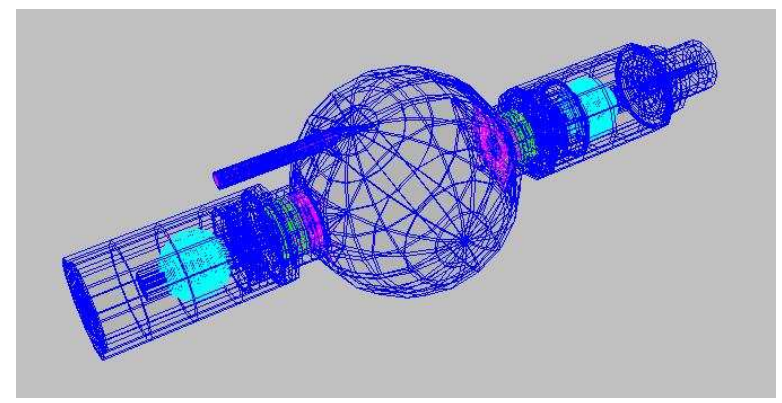

Figure 2. 3D vision of the counter as modelled in MCNPX.

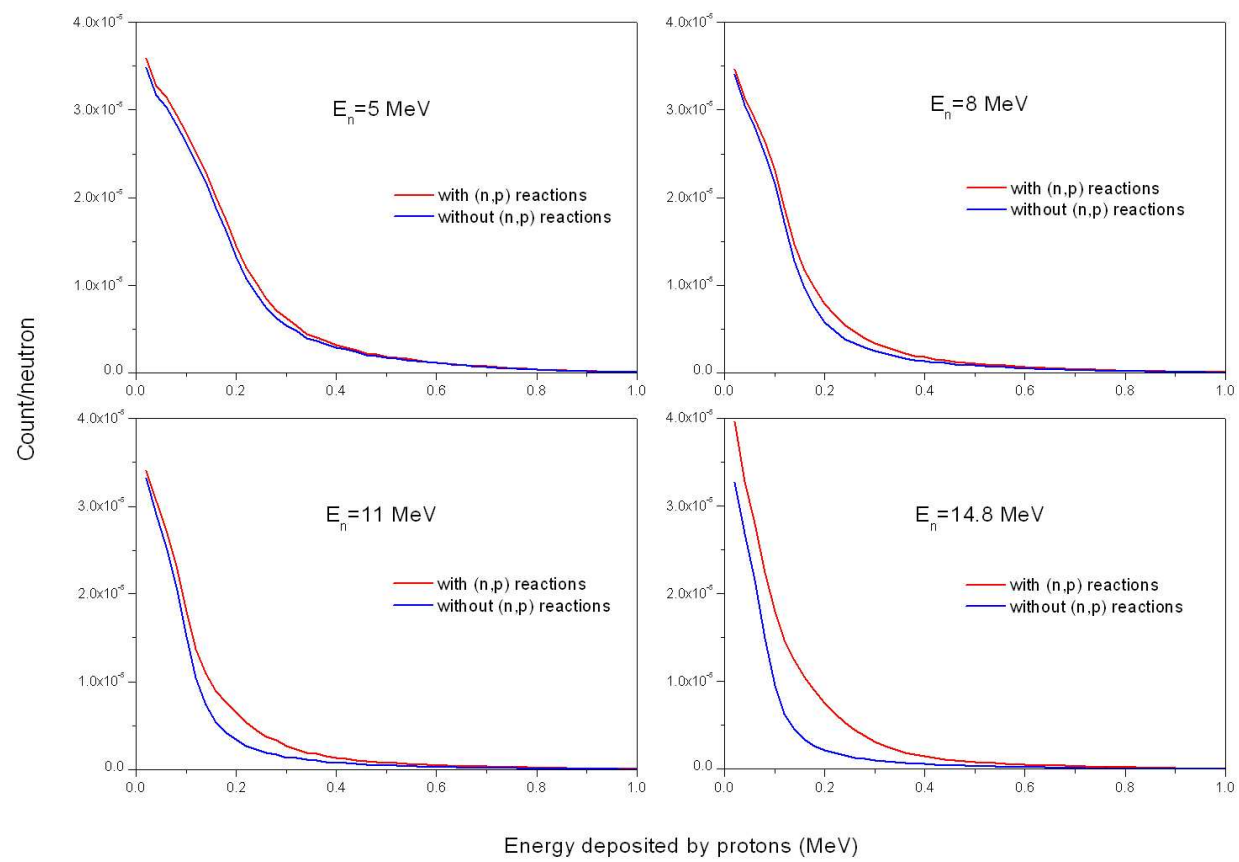

Figure 3. Comparison between the calculated response functions of a spherical counter SP2(H2) $0.3 \mathrm{MPa}$ at 5, 8, 11 and $14.8 \mathrm{MeV}$, with and without (n,p) reactions.

$10 \mathrm{MeV}$, the contribution of the (n,p) reactions to the detector responses depends more on the inside surface of the walls reached by neutrons (i.e. the detector geometry) than on the wall thickness. For neutrons above $15 \mathrm{MeV}$, the maximum proton range exceeds $0.5 \mathrm{~mm}$ and the contribution of the $(\mathrm{n}, \mathrm{p})$ reactions to the detector response increases with the wall thickness.

The energy range of this SP2 counter is between $100 \mathrm{keV}$ and $800 \mathrm{keV}$ [1] Up to now, the $(n, p)$ reactions were generally not included in the response calculation. Following recent developments in the proportional counter response simulation [8], the MCNPX Monte Carlo code has been used in that aim with the ENDF-BVI.8 library. This code allows to describe in detail the counter geometry (as shown in figure 2) and it is possible to take into account the electric field effect inside the detector using the method described in a previous work [8].

The response of this counter has been calculated for 4 energies, respectively 5,8,11 and 14.8 MeV, which were generated from a circular surface, of section equals to the diameter of the detector and, parallel to the horizontal axis of the counter. At each energy, calculations are performed with and without taking into account the $(\mathrm{n}, \mathrm{p})$ reactions and are compared in figure 3. 


\begin{tabular}{|l|c|c|}
\hline & $5 \mathrm{MeV}$ & $14.8 \mathrm{MeV}$ \\
\hline Detector angular position & $0^{\circ}$ & $0^{\circ}$ \\
\hline Incoming deuton energy & $1847.5 \pm 1.6 \mathrm{keV}$ & $208.0 \pm 0.2 \mathrm{keV}$ \\
\hline Beam current & $2.0 \mu \mathrm{A}$ & $3.3 \mu \mathrm{A}$ \\
\hline Target & $\mathrm{TiD}$ & TiT \\
\hline Backing & $\mathrm{Ag}-0.5 \mathrm{~mm}$ & $\mathrm{Al}-0.5 \mathrm{~mm}$ \\
\hline Target name & IRSN04 & IRSN06 \\
\hline Target thickness & $825 \mu \mathrm{g} . \mathrm{cm}-2$ & $796 \mu \mathrm{g} . \mathrm{cm}-2$ \\
\hline Target-counter distance & $48.1 \pm 0.1 \mathrm{~cm}$ & $48.1 \pm 0.1 \mathrm{~cm}$ \\
\hline
\end{tabular}

Table 2. Experimental conditions for the SP2 counter measurements at the AMANDE facility.

As the number of reached reaction thresholds increase with the incident neutron energy, as well as the relative impact of the $(n, p)$ reactions compared to the elastic scattering on hydrogen (figure 1, the discrepancy between the two curves increases logically with the energy, to reach up to $40 \%$ at $14.8 \mathrm{MeV}$, as already observed in a previous work [9]. At $5 \mathrm{MeV}$, only a small difference between both calculations is observed. From this study, it can be concluded that the $(n, p)$ reaction impact has to be considered when neutrons above $5 \mathrm{MeV}$ energy are present in the measured neutron fields. To not take into account these reactions would lead to an important overestimation of the neutron fluence in the detector energy range.

\section{Comparisons between simulations and experimental data}

\subsection{Experimental set-up}

Experimental data were obtained at the AMANDE facility producing mono-energetic field between some $\mathrm{keV}$ up to $20 \mathrm{MeV}$ [6]. The measurements were performed with the SP2 counter at 2 neutron energies: $5 \mathrm{MeV}$ and $14.8 \mathrm{MeV}$, which were produced respectively using the $\mathrm{D}(\mathrm{d}, \mathrm{n})^{3} \mathrm{He}$ and $\mathrm{D}(\mathrm{t}, \mathrm{n})^{4} \mathrm{He}$ reactions. The SP2 counter is well characterized and traceable to the primary reference neutron fields of the PTB in terms of fluence and energy [1]

For this study, the SP2 counter was placed at $48 \mathrm{~cm}$ from the target and at $0^{\circ}$ with respect to the proton beam direction. The reference fluence was determined by the BC501A liquid scintillator of the laboratory [4] with a relative uncertainty of 5\%. The relative fluence delivered during the irradiation was followed by a ${ }^{3} \mathrm{He}$ long counter, in a De Pangher type moderating shielding, situated at 6 meters from the target and at an angle of $20^{\circ}$ with respect to the beam axis. This monitor allowed to normalize the SP2 experimental data to the BC501A reference fluence. The table 2 presents all the experimental conditions to create the mono-energetic fields.

For each measurement carried out with the SP2 counter, an additional measurement with a shadow cone placed between the target and the counter was performed to correct the data from the events due to the scattered neutrons.

\subsection{Comparison with the simulation}

In order to compare the experimental data with the MCNPX simulations, new MCNPX response calculations are performed using the neutron fluence energy distribution calculated by the TARGET code [10], normalized to the reference fluence, as source term. As previously, calculations of the response of the counter are performed with and without taking into account the $(n, p)$ reactions in the detector walls. The results at 5 and $14.8 \mathrm{MeV}$ are shown in figure 4 


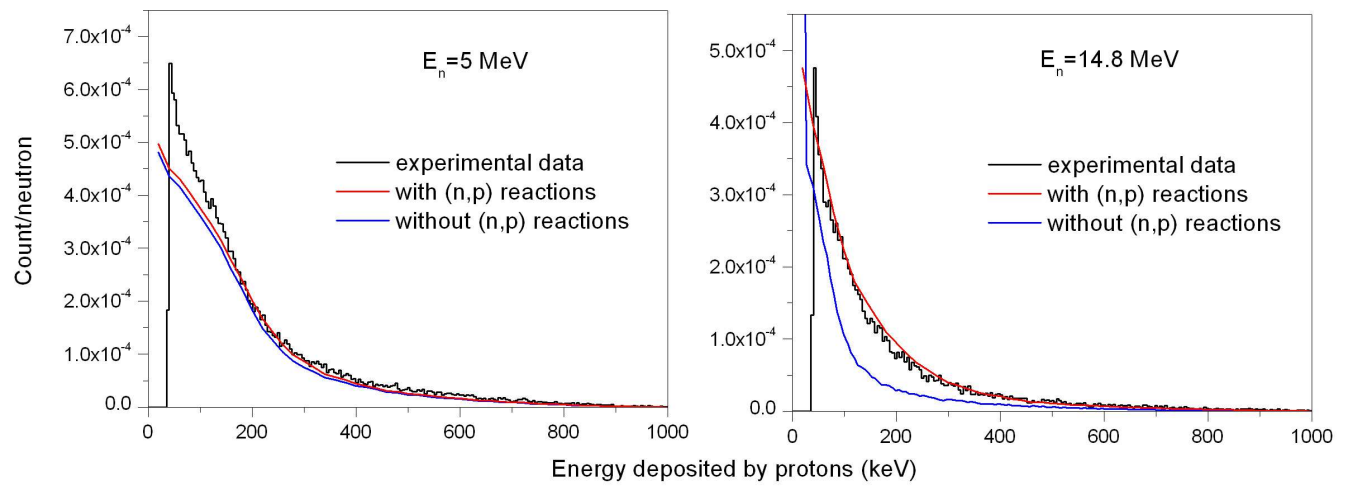

Figure 4. Comparison between the simulated response functions with and without (n,p) reactions and the experimental data obtained at the $5 \mathrm{MeV}$ and $14.8 \mathrm{MeV}$ mono-energetic neutron field.

At $5 \mathrm{MeV}$, both calculations reproduce the measurement above $150 \mathrm{keV}$. Below this value, the discrepancy between the simulation and the experimental data is significant whereas the response of the counter is very good in this energy range [1]. The observed discrepancy is assumed to come from an underestimation of the scattered neutrons by the TARGET code, due to a simplified geometry used to describe the target environment or to the target itself. This point has to be verified in further studies.

At $14.8 \mathrm{MeV}$, a good agreement is observed between the simulation integrating the (n,p) reactions and the measurement. However, the calculation which does not integrate these reactions, underestimates greatly the experimental data. These results shows that the (n,p) reaction contribution to the recoil proton proportional counter response is well described by MCNPX and that this code is suitable to calculate precisely the response matrices of these counters.

\section{Conclusion and outlook}

In this work the impact of the $(n, p)$ reactions on the response of a spherical proton recoil proportional counter was studied. These reactions occur in the detector wall between high energy neutrons and some elements of the stainless steel and their relative contribution to the counter response increases with the incident neutron energy. This study has shown that the (n,p) reaction impact is no more negligible for neutron energies above $5 \mathrm{MeV}$.

The simulation of the detector response with the MCNPX Monte Carlo code including these $(n, p)$ reactions are validated by the measurements performed at mono-energetic neutron fields. The fact to not take into account the $(n, p)$ reactions leads to an underestimation of about $40 \%$ of the detector experimental response at $14.8 \mathrm{MeV}$.

As a conclusion, MCNPX code appears suitable to simulate correctly the response of the proton recoil proportional counter in high energy neutron fields, and then allows the use of these counters for spectrometry in such fields and not only in mono-energetic neutron fields below a few MeV. This study focused on SP2 counters but can be applied to all kind of recoil proton proportional counters as spherical counters, cylindrical counters, or the commercial device ROSPEC. 


\section{Acknowledgments}

The authors would like to thank Dr. Lena Lebreton for her help in providing the reference fluence data at the AMANDE facility.

\section{References}

[1] G. Pichenot, et al., Characterisation of spherical recoil proton proportional counters used for neutron spectrometry, Nucl. Instrum. Meth. A 476 (2002) 165.

[2] K. Knauf and J. Wittstock, neutron spectrometry with proton recoil proportional counters at the research and measurement reactor braunschweig - status of the technique -, Physikalisch Technische Bundesanstalt report PTB-FMRB-114 (1987).

[3] K. Knauf et al., Spectrometry in a mixed neuron-photon field, a field experiment within the framework of the AHE project using a liquid scintillation counter and spherical proton recoil proportional counters, Physikalisch Technische Bundesanstalt report PTB-6.42-97-2 (1998).

[4] V. Gressier et al., Characterization of the IRSN Facility CANEL/T400 Producting realistic neutron fieds for calibration and test purposes, IRSN report SDE/2004-08 (2004).

[5] L.S. Waters, MCNPX - User's manual, Version 2.5.0, Los Alamos National Laboratory report LACP-05-0369 (2005).

[6] V. Gressier, G. Pelcot and J. L. Pochat, New IRSN facilities for neutron production, Nucl. Instrum. Meth. A 505 (2003) 370.

[7] N. Soppera, H. Henriksson, A. Nouri, P. Nagel and E. Dupont, JANIS-2: An improved version of the NEA Java-based nuclear data information system, in proceedings of International Conference on Nuclear Data for Science and Technology, September 26 - October 1, Santa Fe, New Mexico, USA (2004).

[8] R. Babut and V. Gressier, Monte carlo simulation of the response energy distribution of proton recoil proportional counters, in proceedings of International workshop on fast neutron detectors and applications, April, 3-6, 2006 University of Cape Town, South Africa, PoS(FNDA2006)025.

[9] J.S. Petler and M.C. Scott, The effect of charged particules produced in detector walls on the use of proton recoil proportional counters for neutron spectrometry, Nucl. Instrum. Meth. 228 (1985) 425.

[10] D. Schlegel, TARGET - User's manual, Physikalisch Technische Bundesanstalt report PTB-6.4198-1 (1998). 